\title{
Article \\ Proposal of Quick Diagnostic Criteria for Disseminated Intravascular Coagulation
}

\author{
Hideo Wada ${ }^{1, *(\mathbb{D}}$, Akitaka Yamamoto ${ }^{2}$, Masaki Tomida ${ }^{2}$, Yuhuko Ichikawa ${ }^{3}$, Minoru Ezaki ${ }^{3}$, Jun Masuda ${ }^{4}$, \\ Masamichi Yoshida ${ }^{5}$, Shunsuke Fukui ${ }^{6}$, Isao Moritani ${ }^{6}$, Hidekazu Inoue ${ }^{6}$, Katsuya Shiraki ${ }^{6}$, Kei Suzuki ${ }^{7}$, \\ Hiroshi Imai ${ }^{7}$, Motomu Shimaoka ${ }^{8}$ and Hideto Shimpo ${ }^{9}$
}

check for updates

Citation: Wada, H.; Yamamoto, A.; Tomida, M.; Ichikawa, Y.; Ezaki, M.; Masuda, J.; Yoshida, M.; Fukui, S.; Moritani, I.; Inoue, H.; et al. Proposal of Quick Diagnostic Criteria for Disseminated Intravascular Coagulation. J. Clin. Med. 2022, 11, 1028. https://doi.org/10.3390/ jcm11041028

Academic Editor: Andrea Gallamini

Received: 25 January 2022

Accepted: 14 February 2022

Published: 16 February 2022

Publisher's Note: MDPI stays neutral with regard to jurisdictional claims in published maps and institutional affiliations.

Copyright: (C) 2022 by the authors. Licensee MDPI, Basel, Switzerland. This article is an open access article distributed under the terms and conditions of the Creative Commons Attribution (CC BY) license (https:// creativecommons.org/licenses/by/ $4.0 /)$.
1 Department of General and Laboratory Medicine, Mie Prefectural General Medical Center, Yokkaichi 510-0885, Japan

2 Department of Emergency and Critical Care Center, Mie Prefectural General Medical Center, Yokkaichi 510-0885, Japan; akitaka-yamamoto@mie-gmc.jp (A.Y.); st25053@yahoo.co.jp (M.T.)

3 Department of Central Laboratory, Mie Prefectural General Medical Center, Yokkaichi 510-0885, Japan; ichi911239@yahoo.co.jp (Y.I.); ajbyd06188@yahoo.co.jp (M.E.)

4 Department of Cardiovascular Medicine, Mie Prefectural General Medical Center, Yokkaichi 510-0885, Japan; jun-masuda@mie-gmc.jp

5 Department of Respiratory Medicine, Mie Prefectural General Medical Center, Yokkaichi 510-0885, Japan; masamichi-yoshida@mie-gmc.jp

6 Department of Gastroenterology, Mie Prefectural General Medical Center, Yokkaichi 510-0885, Japan; m13092sf@jichi.ac.jp (S.F.); isao-moritani@mie-gmc.jp (I.M.); hidekazu-inoue@mie-gmc.jp (H.I.); katsuya-shiraki@mie-gmc.jp (K.S.)

7 Emergency Critical Care Center, Mie University Graduate School of Medicine, Tsu 514-8507, Japan; keis@med.mie-u.ac.jp (K.S.); hi119@clin.medic.mie-u.ac.jp (H.I.)

8 Department of Molecular Pathobiology and Cell Adhesion Biology, Mie University Graduate School of Medicine, Tsu 514-8507, Japan; motomushimaoka@gmail.com

9 Mie Prefectural General Medical Center, Yokkaichi 510-0885, Japan; hideto-shimpo@mie-gmc.jp

* Correspondence: wadahide@clin.medic.mie-u.ac.jp

Abstract: Background. The diagnostic criteria for disseminated intravascular coagulation (DIC) vary and are complicated and the cut-off values are different. Simple and quick diagnostic criteria for DIC are required in physicians for critical care. Material and methods. Platelet counts, prothrombin time-international normalized ratio (PT-INR) and D-dimer levels were examined in 1293 critical ill patients. Adequate cut-off values of these parameters were determined and a quick DIC score using these biomarkers was proposed. The quick DIC score was evaluated using a receiver operating characteristic (ROC) analysis. Results. Using the Japanese Ministry of Health, Labor and Welfare diagnostic criteria, 70 and 109 patients were diagnosed with DIC and pre-DIC, respectively. The ROC analysis of factors difference between DIC and non-DIC, revealed the following cut-off values: PT-INR, 1.20; platelet count, $12.0 \times 10^{10} / \mathrm{L}$ and D-dimer, $10.0 \mu \mathrm{g} / \mathrm{mL}$. Based on the above results, the quick DIC score system was proposed. All patients with DIC had a quick DIC score of 3, 4 or 5, and $85.3 \%$ of the patients with pre-DIC had a quick DIC score of $\geq 3$ points. All patients with pre-DIC had a score of $\geq 2$ points. In the ROC analysis, the area under the curve was 0.997 for DIC vs. non-DIC, and 0.984 for pre-DIC + DIC vs. non-DIC, and the cut-off value was 3 points for DIC and 2 points for DIC + pre-DIC. The quick DIC scores of non-survivors were significantly higher than those of survivors. Conclusions. The Quick DIC score system is a simple and useful tool that can be used for the diagnosis of DIC and pre-DIC. Further evaluation of the quick DIC score system in a large-scale study is required.

Keywords: DIC; diagnosis; PT-INR; platelet count; D-dimer

\section{Introduction}

Disseminated intravascular coagulation (DIC) is frequently associated with infectious diseases, hematological malignancy, and solid cancer, and causes organ failure and bleed- 
ing symptoms, often resulting in poor outcomes [1,2]. The DIC patients are generally treated for underlying diseases, and those with major bleeding are treated with supplementary therapy from blood products [3]. In Japan, where an early treatment of DIC has been recommended [4], DIC patients have been treated with antithrombin [5] or recombinant thrombomodulin [6]. Therefore, diagnostic criteria for DIC have been established by the Japanese Ministry of Health, Labor and Welfare (JMHLW) [7], the International Society of Thrombosis Haemostasis (ISTH) [8], the Japanese Association for Acute Medicine (JAAM) [9], and the Japanese Society of Thrombosis Hemostasis (JSTH) [10].

These diagnostic criteria for DIC are based on scoring systems that include the platelet counts, prothrombin time (PT), fibrin-related markers, and fibrinogen [11]. The scoring system for DIC may be complicated in practice and fibrin-related markers require standardization [12]. Multiple cut-off values of the parameters are used among the four diagnostic criteria for DIC [11]. In the emergency room, more simple diagnostic criteria are required to facilitate the early treatment of DIC. The recently developed sepsis-induced coagulopathy (SIC) score, which includes PT, platelet count, and the sequential organ failure assessment score, may be a simple and easy score, and may be useful for diagnosing DIC in sepsis patients [13]. The cut-off values of fibrin-related markers have not been established, varying among reports for DIC using ISTH overt-DIC diagnostic criteria [12]. Furthermore, the JAAM diagnostic criteria for DIC are not useful for diagnosing DIC due to non-infectious diseases, and the JSTH diagnostic criteria for DIC are complicated. Therefore, we diagnosed DIC using the JMHLW diagnostic criteria for DIC.

In this study, we determined adequate cut-off values of PT, D-dimer, and platelet count for the diagnosis of DIC and propose a quick DIC score system.

\section{Materials and Methods}

The study population included patients with the following conditions who were managed at Mie Prefectural General Medical Center from 1 September 2019 to 28 December 2020: sepsis $(n=58)$, critical illness without sepsis $(n=152)$, pneumonia $(n=107)$, obstetric disease $(n=16)$, solid cancer $(n=42)$, aneurysm $(n=78)$, other respiratory disease $(n=28)$, peripheral arterial and venous thrombosis (PAVTE, $n=44)$, hematological disorder $(n=41)$, other infection ( $n=77)$, other digestive disorder $(n=87)$, cerebrovascular disorder $(n=176)$, acute coronary syndrome $(n=67)$, other heart disease $(n=94)$, urinary tract disease $(n=125)$, unidentified clinical syndrome $(n=125)$, and other conditions $(n=50)$. DIC was diagnosed using the Japanese Ministry of Health Labor and Welfare criteria for DIC (Supplementary Table S1) [7]. Patients with a DIC scores of $\geq 7$ points, with 5-6 points, and $\leq 4$ points were diagnosed with DIC, pre-DIC, and non-DIC, respectively. Cerebrovascular disorder was diagnosed by computed tomography or magnetic resonance imaging; acute coronary syndrome was diagnosed by coronary angiography, electrocardiography, and the detection of elevated troponin; and PAVTE was diagnosed using computed tomography or venous ultrasound. The study protocol (2019-K9) was approved by the Human Ethics Review Committee of Mie Prefectural General Medical Center, and informed consent was obtained from each participant. This study was carried out in accordance with the principles of the Declaration of Helsinki.

D-dimer was measured using LPIA-Genesis (LSI Medience, Tokyo, Japan), with a STACIA system (LSI Medience). The prothrombin time (PT) international normalized ratio (INR) was measured by a Thromborel S (Sysmex Co., Kobe, Japan) using an automatic coagulation analyzer CS-5100 (Sysmex Co.). The platelet counts were measured using a full-automatic blood cell counter XN-3000 (Sysmex Co.). The median (2.5-97.5 percentile) values of D-dimer, PT-INR, and platelet counts were $0.4 \mu \mathrm{g} / \mathrm{mL} ; 0.1-0.5 \mu \mathrm{g} / \mathrm{mL}, 0.96$; $0.90-1.04$, and $21.9 \times 10^{10} / \mathrm{L} ; 17.9-25.9 \times 10^{10} / \mathrm{L}$, respectively.

\section{Statistical Analyses}

The data are expressed as the median (25-75th percentile). The significance of differences between groups was examined using the Mann-Whitney $U$-test. A multiple 
regression analysis of PT-INR, D-dimer, and platelet counts for diagnosing DIC or pre-DIC vs. non-DIC was conducted. The cut-off values were determined as the point at which the sensitivity curve and specificity curve intersected, and were examined by a receiver operating characteristic (ROC) analysis. $p$ values of $<0.05$ were considered to indicate a statistically significant difference. All of the statistical analyses were performed using the Stat-Flex software program (version 6; Artec Co., Ltd., Osaka, Japan).

\section{Results}

Regarding the evaluation of patients using the JMHLW diagnostic criteria, 70 and 109 patients were diagnosed with DIC and pre-DIC, respectively. Sepsis, critical illness without sepsis, pneumonia, obstetric disease, and solid cancer were frequently associated with DIC (Table 1).

Table 1. Subjects.

\begin{tabular}{ccccc}
\hline Underlying Diseases & Non-DIC & Pre-DIC & DIC & Number of DIC (\%) \\
\hline Sepsis & 26 & 21 & 11 & $11 / 58(19.0 \%)$ \\
Critical illness without sepsis & 95 & 23 & 34 & $34 / 152(22.4 \%)$ \\
Pneumonia & 78 & 17 & 12 & $12 / 107(11.2 \%)$ \\
Obstetric diseases & 13 & 1 & 2 & $2 / 16(12.5 \%)$ \\
Solid cancer & 33 & 6 & 3 & $3 / 42(7.1 \%)$ \\
Aneurysm & 66 & 9 & 3 & $3 / 78(3.8 \%)$ \\
Other respiratory diseases & 24 & 3 & 1 & $1 / 28(3.6 \%)$ \\
Peripheral arterial and venous thrombosis & 39 & 4 & 1 & $1 / 44(2.3 \%)$ \\
Hematological disorders & 40 & 0 & 1 & $1 / 41(2.4 \%)$ \\
Other infections & 64 & 12 & 1 & $1 / 77(1.3 \%)$ \\
Other digestive disorders & 84 & 2 & 1 & $1 / 87(1.1 \%)$ \\
Cerebrovascular disorders & 174 & 2 & 0 & $0 / 176(0 \%)$ \\
Acute coronary syndrome & 66 & 1 & 0 & $0 / 67(0 \%)$ \\
Other heart diseases & 90 & 4 & 0 & $0 / 94(0 \%)$ \\
Urinary tract diseases & 13 & 1 & 0 & $0 / 14(0 \%)$ \\
Unidentified clinical syndrome & 125 & 0 & 0 & $0 / 125(0 \%)$ \\
Others & 51 & 0 & 0 & $0 / 50(0 \%)$ \\
\hline Total & 1114 & 109 & 70 & $70 / 1293(5.4 \%)$ \\
\hline
\end{tabular}

DIC, disseminated intravascular coagulation; Non-DIC, DIC score $\leq 4$; Pre-DIC, DIC score 5 or 6; DIC, DIC score $\geq 7$ using the Japanese Ministry of Health, Labor and Welfare diagnostic criteria.

The PT-INR in patients with DIC (1.61: 1.34-1.96) was significantly higher in comparison to those with pre-DIC (1.24: 1.06-1.38) or non-DIC (1.01: 0.94-1.10), and in patients with pre-DIC than in those with non-DIC (Figure 1a). The results of a multiple regression analysis of PT-INR, D-dimer, and platelet counts for diagnosing DIC or pre-DIC vs. nonDIC are shown in Table 2. Standard $\beta$ value was higher in the order of D-dimer, platelet count, and PT-INR. Regarding the ROC analysis of DIC vs. non-DIC, the adequate cut-off value was 1.20 , the sensitivity and specificity were $88.8 \%$, and the area under the curve (AUC) and negative predictive value (NPV) were 0.934 and 98.9\% (Table 3).

The platelet count was significantly lower in patients with DIC $\left(8.3 \times 10^{10} / \mathrm{L}\right.$ : $\left.5.2-11.4 \times 10^{10} / \mathrm{L}\right)$ than in those with pre-DIC $\left(12.6 \times 10^{10} / \mathrm{L}: 9.2-20.3 \times 10^{10} / \mathrm{L}\right)$ or nonDIC $\left(21.8 \times 10^{10} / \mathrm{L}: 16.9-27.7 \times 10^{10} / \mathrm{L}\right)$, and in those with pre-DIC than in those with non-DIC (Figure 1b). Regarding ROC analysis for DIC vs. non-DIC, the adequate cut-off value was $12.0 \times 10^{10} / \mathrm{L}$, and the sensitivity and specificity were $93.6 \%$ and $80.0 \%$, and the AUC and NPV were 0.925 and $44.1 \%$.

The D-dimer levels in patients with DIC $(25.0 \mu \mathrm{g} / \mathrm{mL}: 16.1-47.8 \mu \mathrm{g} / \mathrm{mL})$ were significantly higher than those in patients with pre-DIC $(17.0 \mu \mathrm{g} / \mathrm{mL}: 8.2-25.7 \mu \mathrm{g} / \mathrm{mL})$ or non-DIC $(1.7 \mu \mathrm{g} / \mathrm{mL}: 0.7-4.8 \mu \mathrm{g} / \mathrm{mL})$, and in those with pre-DIC than in those with nonDIC (Figure 1c). Regarding the ROC analysis of DIC vs. non-DIC, the adequate cut-off value was $10.0 \mu \mathrm{g} / \mathrm{mL}$, the sensitivity and specificity were $93.1 \%$ and $92.5 \%$, respectively, and the AUC and NPV were 0.971 and $99.6 \%$. 


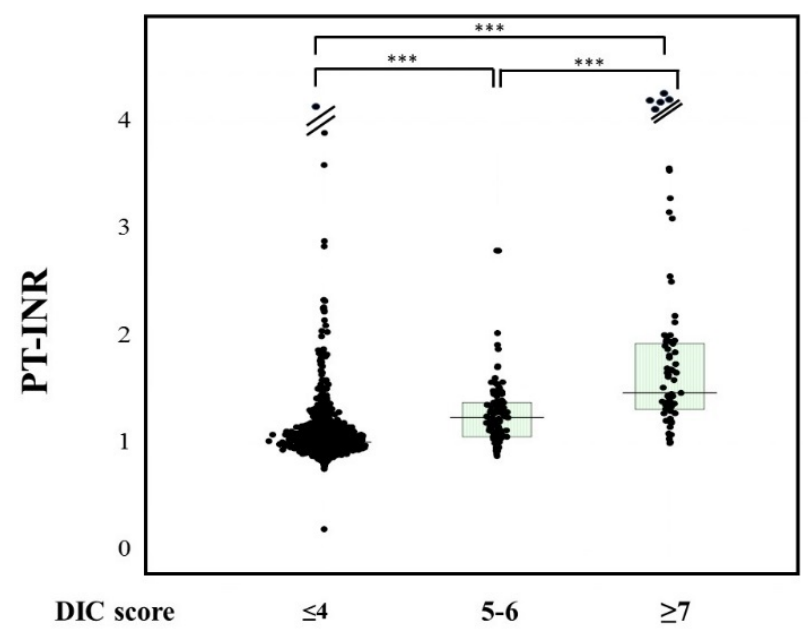

(a)

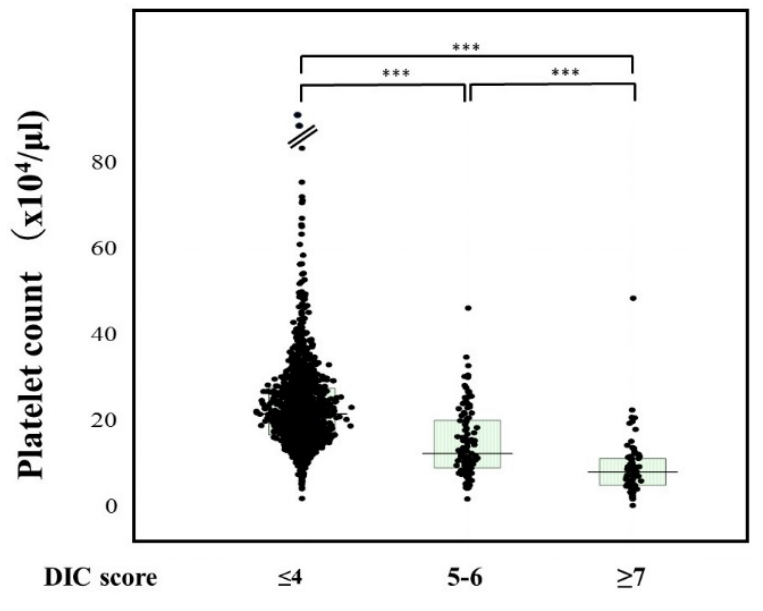

(b)

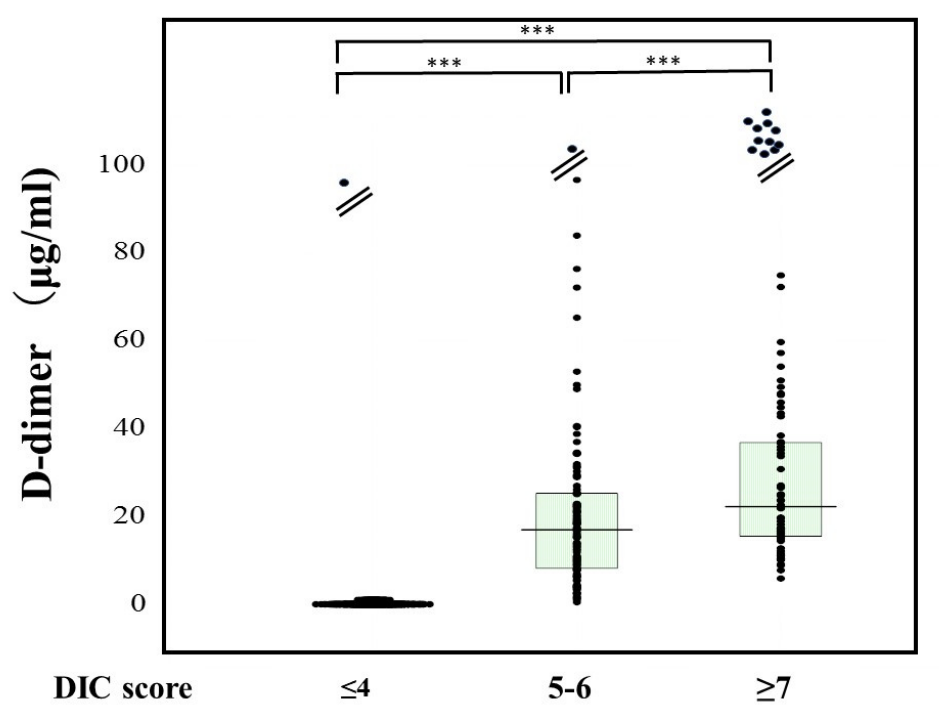

(c)

Figure 1. PT-INR (a), platelet counts (b), and D-dimer (c) DIC, disseminated intravascular coagulation; PT-INR, prothrombin time-international normalized ratio. ${ }^{* * *}, p<0.001$.

Based on the above results, we propose the quick DIC score system (Table 4). When these patients were evaluated using the quick DIC score system, all patients with DIC had a quick DIC score of $\geq 3$ points, $85.3 \%$ of patients with pre-DIC had a score of $\geq 3$ points, and all patients with pre-DIC had a score of $\geq 2$ points (Figure 2). Regarding the ROC analysis (Figure 3a,b), the AUC was 0.997 for DIC vs. non-DIC, and 0.984 for pre-DIC + DIC vs. nonDIC, and the cut-off value was 3 points for DIC and 2 points for DIC + pre-DIC (Table 4). The quick DIC scores of non-survivors (2.0 points: 1.0-3.0 points) were significantly higher than those of survivors (1 point: 0-1.0 points). Regarding the ROC analysis of survival (Figure 3c), the AUC was 0.685 and the adequate cut-off value was 2 points (Table 5). 
Table 2. Results of a multiple regression analysis of PT-INR, D-dimer, and platelet counts for diagnosing DIC or pre-DIC vs. non-DIC.

\begin{tabular}{cccccccc}
\hline & DIC/Pre-DIC & $\boldsymbol{\beta}$ & SE $(\boldsymbol{\beta})$ & $\mathbf{S t d} \boldsymbol{\mathbf { t }}$ & & $\mathbf{N}$ & $\boldsymbol{p}$ \\
\hline \multirow{2}{*}{ PT-INR } & DIC & 0.09643 & 0.01059 & 0.2564 & 9.10465 & 1178 & $<0.000001$ \\
& DIC + Pre-DIC & 0.09596 & 0.01589 & 0.1660 & 6.04085 & 1287 & $<0.000001$ \\
\hline \multirow{2}{*}{ PLT } & DIC & -0.0034 & 0.00046 & -0.2098 & 7.36469 & 1178 & $<0.000001$ \\
& DIC + Pre-DIC & -0.0061 & 0.00068 & -0.2420 & 8.9452 & 1287 & $<0.000001$ \\
\hline \multirow{2}{*}{ D-dimer } & DIC & 0.00379 & 0.00027 & 0.3776 & 13.9969 & 1178 & $<0.000001$ \\
& DIC + Pre-DIC & 0.00501 & 0.00039 & 0.3365 & 12.8174 & 1287 & $<0.000001$ \\
\hline \multirow{2}{*}{ Sex } & DIC & 0.02700 & 0.01144 & 0.0686 & 2.35955 & 1178 & 0.01846 \\
& DIC + Pre-DIC & 0.03496 & 0.01686 & 0.0577 & 2.07386 & 1287 & 0.03829 \\
\hline \multirow{2}{*}{ Age } & DIC & -0.0007 & 0.00029 & -0.0740 & 2.54647 & 1178 & 0.01101 \\
& DIC + Pre-DIC & -0.0005 & 0.00043 & -0.0359 & 1.28869 & 1287 & 0.19774 \\
\hline \multirow{2}{*}{} & &
\end{tabular}

The double correlation coefficient, DIC, $\mathrm{R}=0.57684(p<0.000001)$; Pre-DIC + DIC, $\mathrm{R}=0.51303(p<0.000001)$ DIC, disseminated intravascular coagulation; Non-DIC, DIC score $\leq 4$; Pre-DIC, DIC score 5 or 6; DIC, DIC score $\geq 7$ using the Japanese Ministry of Health, Labor and Welfare diagnostic criteria. PT-INR, prothrombin time-international normalized ratio.

Table 3. ROC analysis of PT-INR, platelet counts, and D-dimer for diagnosing DIC or pre-DIC vs. non-DIC.

\begin{tabular}{cccccccc}
\hline & DIC/Pre-DIC & Cut-Off Value & Sensitivity & Specificity & AUC & NPV & Odds' Ratio \\
\hline \multirow{2}{*}{ PT-INR } & DIC & 1.20 & $88.8 \%$ & $88.0 \%$ & 0.934 & $98.9 \%$ & 45.3 \\
& DIC + Pre-DIC & 1.11 & $77.9 \%$ & $77.9 \%$ & 0.848 & $95.7 \%$ & 12.5 \\
\hline \multirow{2}{*}{ PLT $\left(\times 10^{10} / \mathrm{L}\right)$} & DIC & 12.0 & $93.6 \%$ & $80.0 \%$ & 0.925 & $44.1 \%$ & 58.8 \\
& DIC + Pre-DIC & 16.4 & $76.2 \%$ & $76.2 \%$ & 0.828 & $34.0 \%$ & 10.2 \\
\hline \multirow{2}{*}{ D-dimer $(\mu \mathrm{g} / \mathrm{mL})$} & DIC & 10.0 & $93.1 \%$ & $92.5 \%$ & 0.971 & $99.6 \%$ & 166.4 \\
& DIC + Pre-DIC & 7.8 & $86.4 \%$ & $87.0 \%$ & 0.930 & $97.9 \%$ & 42.5 \\
\hline
\end{tabular}

DIC, disseminated intravascular coagulation; Non-DIC, DIC score $\leq 4$; Pre-DIC, DIC score 5 or 6; DIC, DIC score $\geq 7$ using the Japanese Ministry of Health, Labor and Welfare diagnostic criteria. PT-INR, prothrombin time-international normalized ratio; AUC, area under the curve; NPV, negative predictive value.

Table 4. Quick DIC diagnostic criteria.

\begin{tabular}{ccc}
\hline & Cut-Off Value & Points \\
\hline PT-INR & $\geq 1.2$ & 1 \\
\hline Platelet count & $\leq 12.0 \times 10^{10} / \mathrm{L}$ & 1 \\
\hline D-dimer & $\geq 10.0 \mu \mathrm{g} / \mathrm{mL}$ & 2 \\
\hline Underlying diseases * due to DIC & 1 \\
\hline Total & $\geq 3$ points & Possible DIC \\
\hline
\end{tabular}

$\overline{\text { DIC, disseminated intravascular coagulation; PT-INR, prothrombin time-international normalized ratio; * severe }}$ infections, hematological malignancy, solid cancer, aneurysm, obstetric diseases, critical illness such as trauma, shock and inflammation, multiple organ failure, etc. 


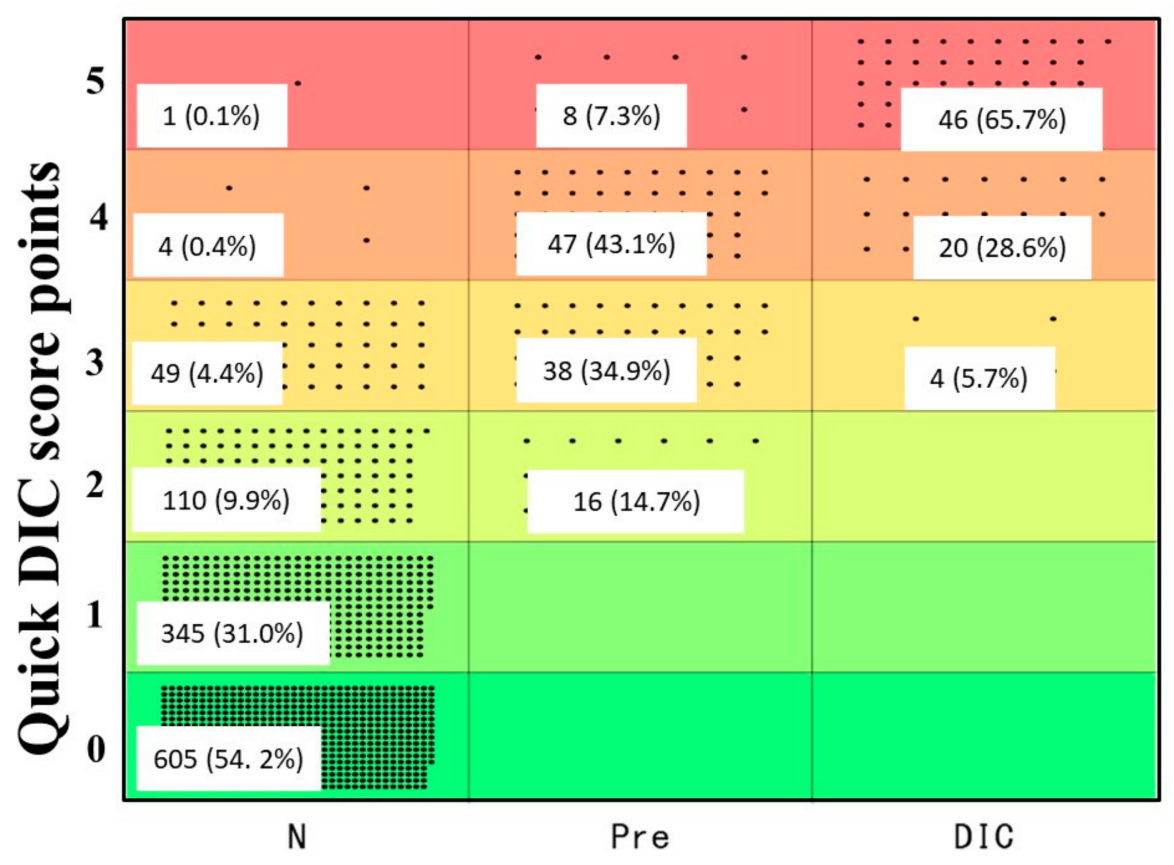

Figure 2. Quick DIC score in patients with non-DIC, pre-DIC, or DIC. DIC, disseminated intravascular coagulation.

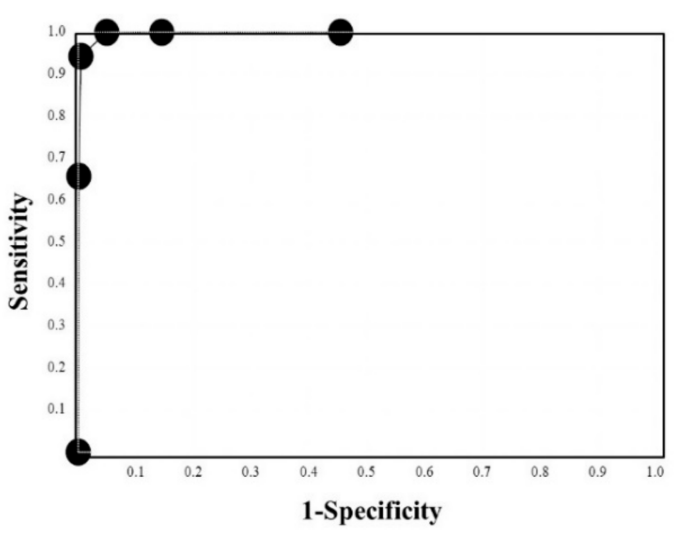

(a)

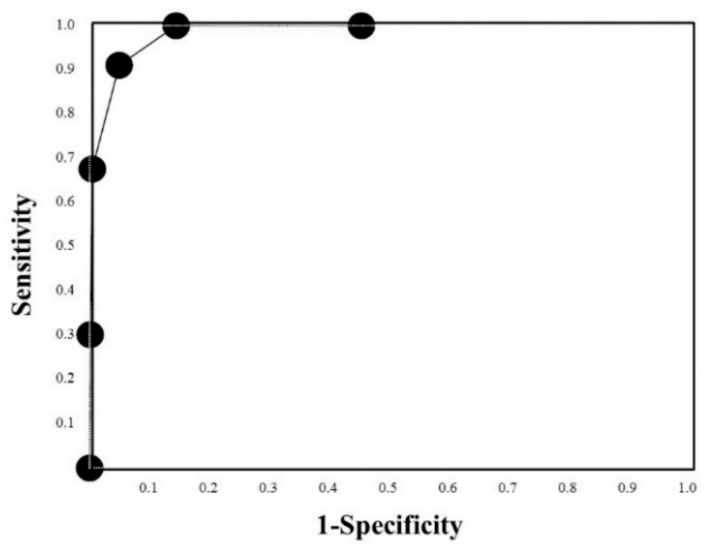

(b)

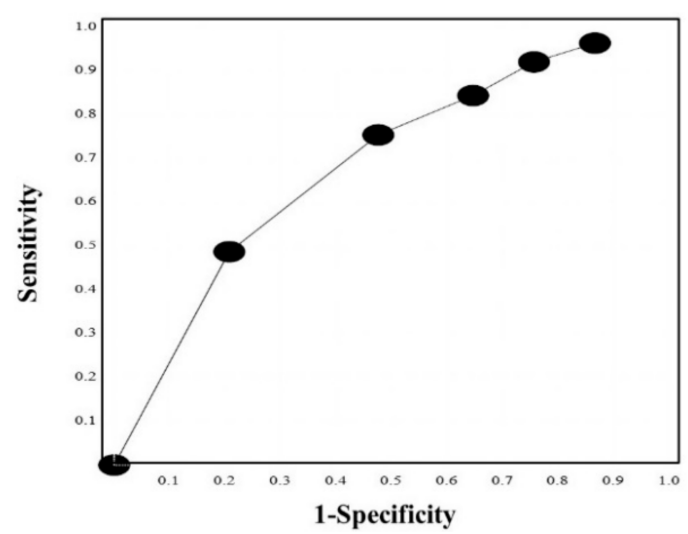

(c)

Figure 3. ROC curve of the quick DIC score for DIC vs. non-DIC (a), DIC and pre-DIC vs. non-DIC (b), or survivor vs. non-survivor (c). ROC, receiver operating characteristic; DIC, disseminated intravascular coagulation; area under the curve, (a) 0.997, (b) 0.984, and (c) 0.685 . 
Table 5. Results of the ROC analysis of the quick DIC score for diagnosing DIC or pre-DIC vs. non-DIC.

\begin{tabular}{|c|c|c|c|c|c|c|}
\hline & Score & Sensitivity & Specificity & PPV & NPV & Likelihood Ratio \\
\hline \multirow{6}{*}{$\begin{array}{c}\text { DIC } \\
\text { AUC, } 0.997 \\
\text { Cut-off value, } 3\end{array}$} & 5 & $65.7 \%$ & $99.9 \%$ & $97.9 \%$ & $94.1 \%$ & 732.1 \\
\hline & 4 & $94.3 \%$ & $99.6 \%$ & $93.0 \%$ & $99.6 \%$ & 210.1 \\
\hline & 3 & $100 \%$ & $95.1 \%$ & $56.0 \%$ & $100 \%$ & 20.3 \\
\hline & 2 & $100 \%$ & $85.5 \%$ & $30.3 \%$ & $100 \%$ & 6.9 \\
\hline & 1 & $100 \%$ & $54.6 \%$ & $12.2 \%$ & $100 \%$ & 2.2 \\
\hline & 0 & $100 \%$ & $0 \%$ & - & - & - \\
\hline \multirow{6}{*}{$\begin{array}{c}\text { DIC + pre-DIC } \\
\text { AUC, } 0.984 \\
\text { Cut-off value, } 2\end{array}$} & 5 & $30.2 \%$ & $99.9 \%$ & $98.2 \%$ & $89.9 \%$ & 480.8 \\
\hline & 4 & $67.6 \%$ & $99.5 \%$ & $96.0 \%$ & $95.0 \%$ & 462.7 \\
\hline & 3 & $91.1 \%$ & $95.1 \%$ & $74.8 \%$ & $98.5 \%$ & 18.4 \\
\hline & 2 & $100 \%$ & $85.5 \%$ & $52.6 \%$ & $100 \%$ & 6.9 \\
\hline & 1 & $100 \%$ & $54.6 \%$ & $26.1 \%$ & $100 \%$ & 2.2 \\
\hline & 0 & $100 \%$ & $0 \%$ & - & - & - \\
\hline
\end{tabular}

DIC, disseminated intravascular coagulation; PPV, positive predictive value; NPV, negative predictive value.

\section{Discussion}

About five percent of patients, especially those with critical illness (e.g., sepsis, pneumonia, obstetric disease, solid cancer, and aneurysms) developed DIC in this study. Therefore, we propose that severe infections, hematological malignancy, solid cancer, aneurysm, obstetric diseases, and critical illness (e.g., trauma, shock and inflammation, multiple organ failure, etc.) are included as underlying diseases due to DIC in the quick DIC scoring system (this is similar to the underlying diseases of DIC in the JMHLW DIC diagnostic criteria for DIC) [7]. These diseases are also generally recognized as underlying diseases of DIC $[8,9]$.

In the ROC analysis, the PT-INR, platelet counts, and D-dimer levels showed high AUC values, suggesting that these parameters are useful for the diagnosis of DIC or pre-DIC. Although D-dimer has the highest AUC and Std $\beta$ among the three parameters, it requires standardization [12] and the SIC scoring system does not include D-dimer [13]. Although the PT-INR has a high AUC and NPV, it reflects the liver function and anticoagulation therapy [14]. Although the platelet count is the most common parameter assessed DIC and has a high AUC, it was found to have a low NPV in this study, suggesting that thrombocytopenia is frequently observed in patients without DIC [15]. Finally, there are no perfect parameters for diagnosing DIC. Therefore, we propose our quick DIC scoring system, which uses a combination of three parameters (e.g., PT-INR, platelet count, and D-dimer) to diagnose DIC. In addition, we propose a single cut-off value for each parameter instead of multiple cut-off values (as used in the JMHLW [7], ISTH [8], JAAM [9], and JSTH [10] diagnostic criteria for DIC), which results in a simple and quick scoring system that can be used for the diagnosis of DIC in the critical care setting.

Regarding the evaluation of the diagnosis of DIC using the quick DIC score system, $100 \%$ of the patients with DIC and $85.3 \%$ of the patients with pre-DIC who were diagnosed using JMHLW diagnostic criteria in this study were diagnosed with possible DIC using the quick DIC score system, which showed a markedly high AUC for the diagnosis of DIC. Therefore, large-scale studies are required to confirm the usefulness of this quick DIC score system for the diagnosis of DIC and pre-DIC. Regarding the survival of patients assessed using the quick DIC score system, approximately $50 \%$ of the patients diagnosed with possible DIC died, suggesting that the quick DIC score may predict poor outcomes as DIC patients still show poor outcomes and DIC is one of the most important causes of death in critical ill patients [3,16].

Finally, the most important point concerning the quick DIC system is its use as a reference point of underlying diseases due to the onset of DIC. The second point is a single 
cut-off value of PT and D-dimer which has a high NPV for diagnosing DIC. The third point is a single cut-off value of platelet counts, which has a high sensitivity for diagnosing DIC.

\section{Conclusions}

The quick DIC score, which is determined based on underlying diseases, the PT-INR, platelet count, and D-dimer levels, was able to diagnose DIC and predict poor outcomes.

Supplementary Materials: The following supporting information can be downloaded at: https: / /www.mdpi.com/article/10.3390/jcm11041028/s1, Table S1: Modified diagnostic criteria for DIC established by Japanese Ministry of Health, Labor and Welfare.

Author Contributions: Methodology, Y.I. and M.E.; data curation, M.T. and S.F.; original draft preparation, H.W.; writing-review and editing, A.Y. and K.S. (Kei Suzuki); investigation, J.M., M.Y., I.M. and H.I. (Hidekazu Inoue); validation, H.I. (Hiroshi Imai); project administration, M.S.; funding acquisition, K.S. (Katsuya Shiraki); supervision, H.S. All authors have read and agreed to the published version of the manuscript.

Funding: This research was funded by a grant-in-aid (H30-015) from the Ministry of Health, Labor and Welfare of Japan.

Institutional Review Board Statement: The study protocol (O-0057) was approved by the Human Ethics Review committees of Mie Prefectural General Medical Center, and informed consent was obtained from each patient.

Informed Consent Statement: Informed consent was obtained from all subjects involved in the study.

Data Availability Statement: The data presented in this study are available on request from the corresponding author. The data are not publicly available due to privacy restrictions.

Acknowledgments: We would like thank the physicians and the laboratory technicians (Chief technicians, Shinya Hiromori and Motoko Tanaka) for measuring the laboratory biomarkers at Mie Prefectural General Medical Center Hospital.

Conflicts of Interest: D-dimer kits were supplied by LSI Medience. The authors declare no other conflict of interest.

\section{References}

1. Iba, T.; Levi, M.; Levy, J.H. Sepsis-Induced Coagulopathy and Disseminated Intravascular Coagulation. Semin. Thromb. Hemost. 2020, 46, 89-95. [PubMed]

2. Wada, H.; Matsumoto, T.; Suzuki, K.; Imai, H.; Katayama, N.; Iba, T.; Matsumoto, M. Differences and similarities between disseminated intravascular coagulation and thrombotic microangiopathy. Thromb. J. 2018, 16, 14. [CrossRef] [PubMed]

3. Wada, H.; Thachil, J.; Di Nisio, M.; Mathew, P.; Kurosawa, S.; Gando, S.; Kim, H.K.; Nielsen, J.D.; Dempfle, C.E.; Levi, M.; et al. The Scientific Standardization Committee on DIC of the International Society on Thrombosis Haemostasis: Guidance for diagnosis and treatment of DIC from harmonization of the recommendations from three guidelines. J. Thromb. Haemost. 2013, 11, 761-767. [CrossRef] [PubMed]

4. Wada, H.; Wakita, Y.; Nakase, T.; Shimura, M.; Hiyoyama, K.; Nagaya, S.; Mori, Y.; Shiku, H. Outcome of disseminated intravascular coagulation in relation to the score when treatment was begun. Thromb. Haemost. 1995, 74, 848-852. [CrossRef] [PubMed]

5. Kuroda, H.; Masuda, Y. Comparison of Protective Effects of Recombinant Antithrombin Gamma and Plasma-Derived Antithrombin on Sepsis-Induced Disseminated Intravascular Coagulation and Multiple Organ Failure. Clin. Appl. Thromb. Hemost. 2020, 26, 1076029620981630. [CrossRef] [PubMed]

6. Akatsuka, M.; Masuda, Y.; Tatsumi, H.; Sonoda, T. The effect of recombinant human soluble thrombomodulin on renal function and mortality in septic disseminated intravascular coagulation patients with acute kidney injury: A retrospective study. $J$. Intensive Care 2020, 8, 94. [CrossRef] [PubMed]

7. Kobayashi, N.; Maegawa, T.; Takada, M.; Tanaka, H.; Gonmori, H. Criteria for diagnosis of DIC based on the analysis of clinical and laboratory findings in 345 DIC patients collected by the Research Committee on DIC in Japan. Bibl. Haemotol. 1983, 49, 265-275.

8. Taylor, F.B., Jr.; Toh, C.H.; Hoots, W.K.; Wada, H.; Levi, M. Towards definition, clinical and laboratory criteria, and a scoring system for disseminated intravascular coagulation-On behalf of the Scientific Subcommittee on disseminated intravascular coagulation (DIC) of the International Society on Thrombosis and Haemostasis (ISTH). Thromb. Haemost. 2001, 86, 1327-1330. [PubMed] 
9. Gando, S.; Iba, T.; Eguchi, Y.; Ohtomo, Y.; Okamoto, K.; Koseki, K.; Mayumi, T.; Murata, A.; Ikeda, T.; Ishikura, H.; et al. A multicenter, prospective validation of disseminated intravascular coagulation diagnostic criteria for critically ill patients: Comparing current criteria. Crit. Care Med. 2006, 34, 625-631. [CrossRef] [PubMed]

10. Wada, H.; Takahashi, H.; Uchiyama, T.; Eguchi, Y.; Okamoto, K.; Kawasugi, K.; Madoiwa, S.; Asakura, H. DIC subcommittee of the Japanese Society on Thrombosis and Hemostasis: The approval of revised diagnostic criteria for DIC from the Japanese Society on Thrombosis and Hemostasis. Thromb. J. 2017, 15, 17. [CrossRef] [PubMed]

11. Wada, H.; Matsumoto, T.; Yamashita, Y.; Hatada, T. Disseminated Intravascular Coagulation: Testing and Diagnosis. Clin. Chim. Acta 2014, 436, 130-134. [CrossRef] [PubMed]

12. Suzuki, K.; Wada, H.; Imai, H.; Iba, T.; Thachil, J.; Toh, C.H. Subcommittee on Disseminated Intravascular Coagulation. A re-evaluation of the D-dimer cut-off value for making a diagnosis according to the ISTH overt-DIC diagnostic criteria: Communication from the SSC of the ISTH. J. Thromb. Haemost. 2018, 16, 1442-1444. [CrossRef] [PubMed]

13. Iba, T.; Levy, J.H.; Raj, A.; Warkentin, T.E. Advance in the Management of Sepsis-Induced Coagulopathy and Disseminated Intravascular Coagulation. J. Clin. Med. 2019, 8, 728. [CrossRef] [PubMed]

14. Wada, H.; Shiraki, K.; Shimaoka, M. The prothrombin time ratio is not a more effective marker for evaluating sepsis-induced coagulopathy than fibrin-related markers. J. Thromb. Hemost. 2020, 18, 1506-1507. [CrossRef] [PubMed]

15. Swain, F.; Bird, R. How I approach new onset thrombocytopenia. Platelets 2020, 31, 285-290. [CrossRef] [PubMed]

16. Yamamoto, A.; Wada, H.; Ichkawa, Y.; Tanaka, M.; Tashiro, H.; Shiraki, K.; Shimpo, H.; Yamashita, Y.; Mastumoto, T.; Shimaoka, M.; et al. Soluble C-Type Lectin-Like Receptor 2 Is a Biomarker for Disseminated intravascular Coagulation. J. Clin. Med. 2021, 10, 2860. [CrossRef] [PubMed] 